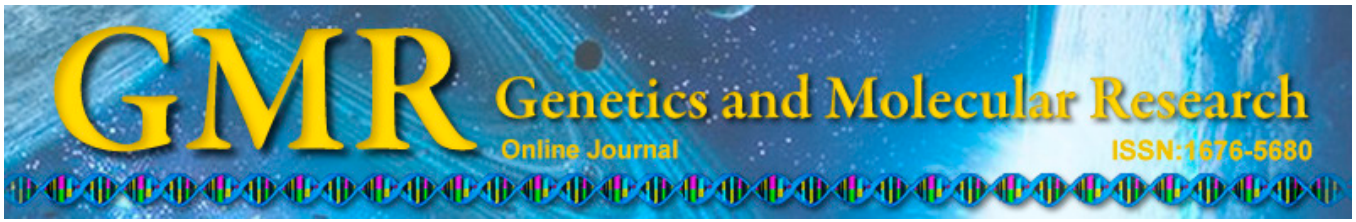

\title{
Effects of goal-directed fluid therapy with different lactated Ringer's: hydroxyethyl starch ratios in hemorrhagic shock dogs
}

\author{
J.P. Tao ${ }^{1}$, Q.Q. Huang ${ }^{2}$, H.Q. Huang ${ }^{2}$, J.J. Yang ${ }^{1}$, M. Shi' ${ }^{1}$ Y. Zhou ${ }^{1}$, \\ L.J. Wan' ${ }^{2}$ C. Zhou ${ }^{1}$, Y.J. Ou ${ }^{1}$, Y.Y. Tong ${ }^{3}$, D.G. Yang ${ }^{4}$ and Y.Y. Si ${ }^{1}$ \\ ${ }^{1}$ Department of Anesthesiology, \\ Second Affiliated Hospital of Kunming Medical University, Kunming, China \\ ${ }^{2}$ Department of Intensive Care Unit, \\ Second Affiliated Hospital of Kunming Medical University, Kunming, China \\ ${ }^{3}$ Department of Radiology, \\ Second Affiliated Hospital of Kunming Medical University, Kunming, China \\ ${ }^{4}$ Department of Cardiovascular Surgery, \\ Second Affiliated Hospital of Kunming Medical University, Kunming, China \\ Corresponding author: Y.Y. Si \\ E-mail: yongyusi@126.com
}

Genet. Mol. Res. 14 (2): 6649-6663 (2015)

Received June 9, 2014

Accepted October 28, 2014

Published June 18, 2015

DOI http://dx.doi.org/10.4238/2015.June.18.8

ABSTRACT. The effects of goal-directed fluid therapy, with lactated Ringer's (LR) and 6\% hydroxyethyl starch (HES) solution, on hemorrhagic shock dogs are unknown. We aimed to determine the optimal LR: HES ratio for the resuscitation of hemorrhagic shock dogs. Hemorrhagic shock was induced in 40 ventilated dogs by drawing an estimated $60 \%$ blood volume. The animals were randomly divided into five groups $(\mathrm{N}=8)$ according to the LR: HES ratio of the resuscitation fluid (3:1, 2:1, 1:1, 1:2, and 1:3), and were then resuscitated for $24 \mathrm{~h}$ to reach the stroke volume variation (SVV) and hemoglobin (Hb) goals by fluid infusion and autologous blood perfusion. The extravascular lung water index (EVLWI), $\mathrm{pH}$, partial pressure of oxygen $\left(\mathrm{PaO}_{2}\right)$, base 
excess (BE), sodium, chloride, $\mathrm{Hb}$ and creatinine clearance (Clearcrea) were checked after $24 \mathrm{~h}$ (R24). The EVLWI of the 3:1 group at R24 were higher than that of the 1:3 group and the baseline value $(\mathrm{P}<0.05)$, whereas the $\mathrm{PaO}_{2}$ was lower $(\mathrm{P}<0.05)$. In contrast to the $3: 1$ group at R24 and baseline, plasma chloride and sodium in the 1:3 and 1:2 groups increased; however, $\mathrm{pH}, \mathrm{BE}$, and Clearcrea decreased $(\mathrm{P}<0.05)$. No significant differences were found in the 1:1 and 2:1 groups at R24 compared with baseline $(\mathrm{P}>0.05)$. Resuscitation with LR and HES at 2:1 and 1:1 ratios are superior in maintaining the acid-base, electrolyte, and lung water balances as well as renal function in hemorrhagic shock dogs than at ratios of $3: 1,1: 2$, and1:3.

Key words: Hemorrhage; Hypovolemic shock; Plasma substitutes; Fluid therapy

\section{INTRODUCTION}

Hemorrhagic shock accounts for about $40 \%$ of civilian deaths and $50 \%$ of military deaths worldwide (Holcomb et al., 2007). Resuscitation of persons with hemorrhagic shock by means of fluid infusion is a routine intervention; however, it also presents a particular challenge to anesthesiologists and critical care physicians. Aggressive iv fluid resuscitation, to restore critical tissue perfusion and oxygen delivery, remains a critical component of treatment of hemorrhagic shock; however, there is an ongoing debate about the best type of solution to be used (i.e., crystalloid vs colloid infusion) (Haas et al., 2008).

Large amounts of crystalloids may cause pulmonary edema and acidosis in hemorrhagic shock patients. However, infusion of excessive colloids such as hydroxyethyl starch (HES) may impair the renal function (Cabrales et al., 2007).

Although published data demonstrate that intraoperative fluid resuscitation with a combination of crystalloids and colloids are superior to administration of either crystalloid or colloid alone, with regard to tissue edema, coagulopathy, and postoperative outcomes (Moretti et al., 2003), the appropriate crystalloid: colloid ratio is still largely unknown.

HES is frequently used as a colloid solution to replace blood volume losses in hypovolemic shock and critically ill patients, as it has been shown to have longer-lasting effects on hemodynamic support owing to its longevity within the intravascular space (Warren and Durieux, 1997; European Medicines Agency, 2013).

Recent studies (Myburgh et al., 2012; Perner et al., 2012) compared the effects of HES with those of crystalloids in critically ill patients, and found that patients with severe sepsis treated with HES were at a greater risk of kidney injury and mortality. The European Medicines Agency's Pharmacovigilance Risk Assessment Committee was therefore requested to assess the available evidence to determine the risk-benefit balance of using HES infusion solutions in the management of hypovolemic shock (European Medicines Agency, 2013).

A goal-directed fluid therapy, which aims to achieve maximal cardiac output and stroke volume while avoiding fluid overload, is associated with reductions in mortality, postoperative complications, and length of hospital stay compared with fluid management guided with traditional physiologic targets [heart rate (HR), blood pressure (BP), central venous pressure, urinary output, arterial oxygenation, and pH] (Pearse et al., 2005; Scheeren et al., 2013). 
However, few studies have focused on the effects of goal-directed fluid therapy with different lactated Ringer's (LR): HES ratios on the acid-base and electrolyte balances, extravascular lung water (EVLW), and renal function in hemorrhagic shock patients. The main aim of this study is to investigate the effects of goal-directed fluid therapy with different LR: HES ratios (1:3, 1:2, 1:1, $2: 1$, and $3: 1$ ) on the above-mentioned parameters in a canine model of hemorrhagic shock. We hypothesized that resuscitation with fluid having different LR: HES ratios would likely result in different effects on the acid-base and electrolyte balances, EVLW, and renal function. An additional aim was to determine the optimal LR: HES ratio for the treatment of hemorrhagic shock dogs.

\section{MATERIAL AND METHODS}

\section{Animal surgical preparation}

The study design was a randomized, controlled trial in a canine model. This study was carried out in accordance with the standard ARRIVE guidelines. The animal use protocol has been reviewed and approved by the Institutional Animal Care and Use Committee of Kunming Medical University. Forty healthy mongrel dogs (weight $15-17 \mathrm{~kg}$ ) were fasted for $12 \mathrm{~h}$ with free access to water. Anesthesia was induced with $10 \mathrm{mg} / \mathrm{kg}$ ketamine (Yingchun; Fujian Gutian Pharmaceutical Co., Ltd., Gutian, Fujian, China) and $i m$ and $i v$ administration of $30 \mathrm{mg} / \mathrm{kg}$ pentobarbital sodium (Tianjin Pharmaceutical Co., Ltd., Tianjin, China). After intubation with a cuffed endotracheal tube (internal diameter, $6.5 \mathrm{~mm}$ ), the dogs were ventilated in a volume-controlled mode (Draeger; Fabius TIRO, Lübeck, Germany) with a tidal volume of $10 \mathrm{~mL} / \mathrm{kg}$, an inspiratory:expiratory ratio of 1:1.5, and an $\mathrm{FiO}_{2}$ of 0.35 . A compact anesthesia monitor (S/5 Datex-Omeda; GE Healthcare, Helsinki, Finland) was attached to the endotracheal tube for continuous measurement of the end-tidal carbon dioxide concentration $\left(\right.$ ET- $\left.-\mathrm{CO}_{2}\right)$ and tidal volume throughout the study. The respiratory rate (25-35 breaths/min) was adjusted to maintain ET- $\mathrm{CO}_{2}$ between 40 and $45 \mathrm{mmHg}$. Anesthesia was maintained with $1.2 \%$ isoflurane (Aerrane; Baxter, Guayama, Puerto Rico) and $2 \mu \mathrm{g} \cdot \mathrm{kg}^{-1} \cdot \mathrm{min}^{-1}$ vecuronium (Xianlin; Zhejiang Xianju Pharmaceutical Co., Ltd., Xianju, China). Immediately after the commencement of mechanical ventilation, all animals received $0.5 \mathrm{~g}$ cefoperazone sodium (Dahua Pharmaceutical Co., Ltd., Wuhan, China) intravenously as antibiotic prophylaxis. The animals were placed in the supine position. A catheter for canines (Buster; Eickemeyer Ltd; Copenhagen, Denmark) was inserted into the urinary bladder of the dogs for the drainage of urine and monitoring of urine output. The core temperature was measured from the thermistor in the PiCCO catheter and maintained at $38 \pm 0.5^{\circ} \mathrm{C}$ with a heating blanket.

A triple-lumen central venous (CV) catheter (Arrow, Asheboro, NC, USA) was inserted percutaneously through the right external jugular vein for blood sampling, blood withdrawal, blood infusion, and fluid resuscitation. A 4F thermistor-tipped catheter for thermodilution and pulse contour analysis (PVPK 2014 L08; Pulsion Medical Systems AG, Munich, Germany) was inserted percutaneously into the femoral artery and connected to a PiCCO monitoring system (Pulsion) for continuous BP monitoring, arterial blood sampling, and transpulmonary thermodilution determinations of stroke volume variation (SVV), cardiac output (CO), and EVLW. Body surface area was calculated according to the formula $k \times \mathrm{BW}^{2 / 3}$ (where the $k$ value was 0.112 for dogs) (Guyton et al., 1973), and used for the determination of the cardiac index (CI) and EVLW index (EVLWI) with the aid of the PiCCO monitor. CO was calibrated in triplicate by injecting $10 \mathrm{~mL} 0.9 \%$ normal saline at a temperature of $2-5^{\circ} \mathrm{C}$ through the $\mathrm{CV}$ 
catheter (anesthesia and instrumentation period; time point 1 ; total of $1 \mathrm{~h}$ ).

\section{Experimental protocol}

The study's time course is shown in Figure 1. After the surgical procedures, baseline measurements were obtained after $30 \mathrm{~min}$ of stable hemodynamic condition (equilibration period; time point 2; baseline; total of $0.5 \mathrm{~h}$ ). After stabilization, hemorrhagic shock was induced by withdrawing $60 \%$ of the estimated total blood volume ( $7 \%$ of body weight) through the $\mathrm{CV}$ catheter within $30 \mathrm{~min}\left(1.2 \mathrm{~mL} \cdot \mathrm{kg}^{-1} \cdot \mathrm{min}^{-1}\right)$ (bleeding period; time point 3 ; shock 0 ; total of 0.5 h). The shed blood was collected rapidly into sterile blood bank bags (three-bag collection set; citrate-phosphate-dextrose-adenine; Weigao Products, Weihai, China).

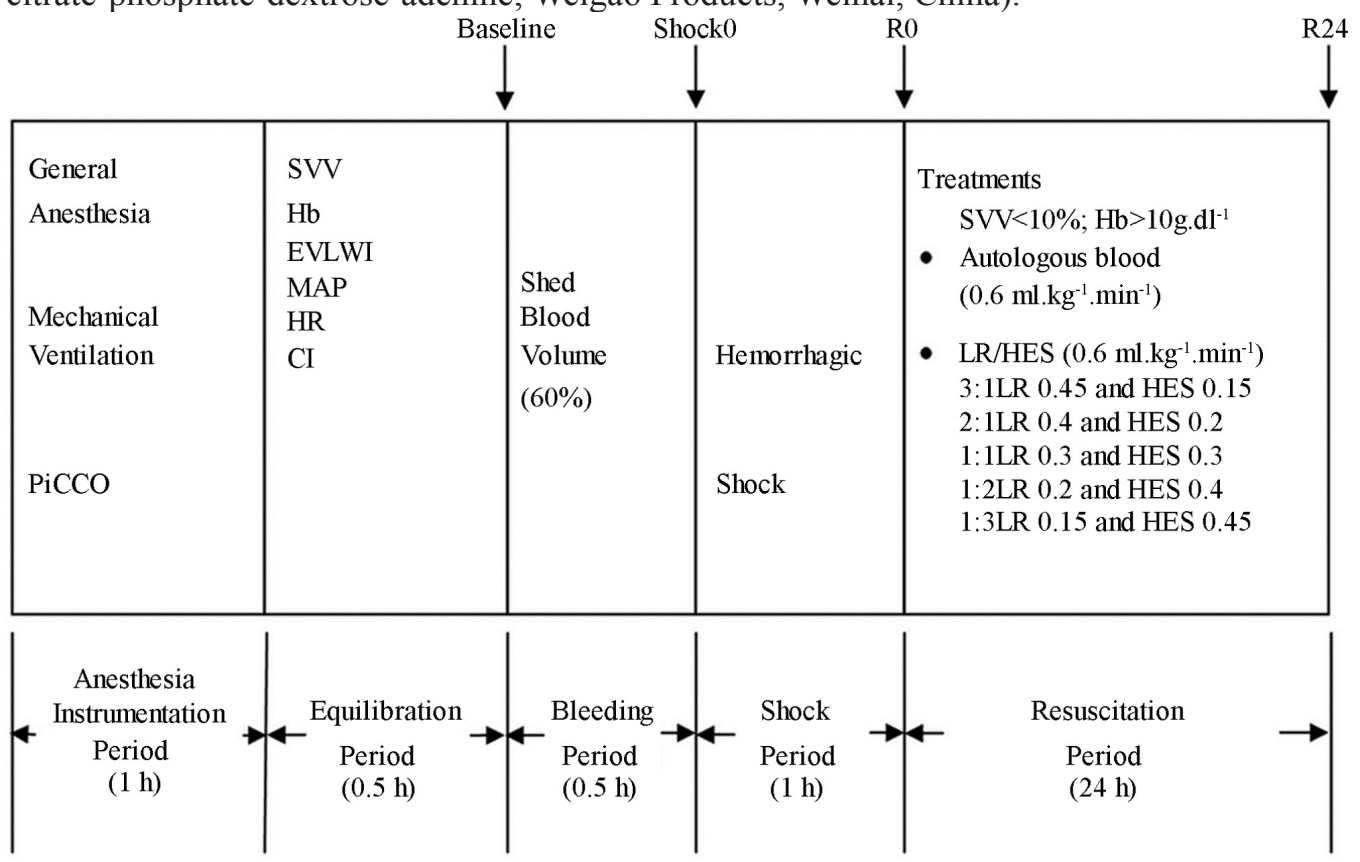

Figure 1. Experimental time course.

\section{Fluid treatment groups}

In this study, HES 130/0.4 (Voluven; Fresenius Kabi, Graz, Austria) and LR solution (Baxter, Guangzhou, China) were used as resuscitation fluids. SVV and hemoglobin ( $\mathrm{Hb})$ were considered the resuscitation goals.

One hour after hemorrhagic shock (shock period; time point 4; R0; total of $1 \mathrm{~h}$ ), dogs were randomly divided into five groups $(\mathrm{N}=8)$ according to the different LR: HES ratios of the resuscitation fluid: $3: 1,2: 1,1: 1,1: 2$, and $1: 3$ groups. The animals then received either fluid of different LR: HES ratios or $0.6 \mathrm{~mL} \cdot \mathrm{kg}^{-1} \cdot \mathrm{min}^{-1}$ autologous blood to reach the $\mathrm{SVV}$ and $\mathrm{Hb}$ goals. The aim was to maintain the SVV $<10 \%$ and $\mathrm{Hb}>10 \mathrm{~g} / \mathrm{dL}$ by using the resuscitation fluid and autologous blood (resuscitation period; time point 5; total of $24 \mathrm{~h}$ ).

LR and HES were infused at the following rates for each group: 3:1 group, 0.45 
$\mathrm{mL} \cdot \mathrm{kg}^{-1} \cdot \mathrm{min}^{-1} \mathrm{LR}$ and $0.15 \mathrm{~mL} \cdot \mathrm{kg}^{-1} \cdot \mathrm{min}^{-1}$ HES; $2: 1$ group, $0.4 \mathrm{~mL} \cdot \mathrm{kg}^{-1} \cdot \mathrm{min}^{-1} \mathrm{LR}$ and 0.2 $\mathrm{mL} \cdot \mathrm{kg}^{-1} \cdot \mathrm{min}^{-1} \mathrm{HES}$; 1:1 group, $0.3 \mathrm{~mL} \cdot \mathrm{kg}^{-1} \cdot \mathrm{min}^{-1} \mathrm{LR}$ and $0.3 \mathrm{~mL} \cdot \mathrm{kg}^{-1} \cdot \mathrm{min}^{-1} \mathrm{HES} ; 1: 2$ group, $0.2 \mathrm{~mL} \cdot \mathrm{kg}^{-1} \cdot \mathrm{min}^{-1} \mathrm{LR}$ and $0.4 \mathrm{~mL} \cdot \mathrm{kg}^{-1} \cdot \mathrm{min}^{-1} \mathrm{HES}$; and $1: 3$ group, $0.15 \mathrm{~mL} \cdot \mathrm{kg}^{-1} \cdot \mathrm{min}^{-1} \mathrm{LR}$ and $0.45 \mathrm{~mL} \cdot \mathrm{kg}^{-1} \cdot \mathrm{min}^{-1} \mathrm{HES}$. Then, the rate of fluid infusion was adjusted to $0.06 \mathrm{~mL} \cdot \mathrm{kg}^{-1} \cdot \mathrm{min}^{-1}$ and maintained at this constant rate when the SVV decreased to $<10 \%$. The Hb concentration was measured spectrophotometrically by using a single drop of blood (HemoCue $\mathrm{Hb} 201+$ analyzer, Angelholm, Sweden). Retransfusion of the shed blood was stopped when the $\mathrm{Hb}$ level increased to $>10 \mathrm{~g} / \mathrm{dL}$ (maintained between 10 and $11 \mathrm{~g} / \mathrm{dL}$ ). $\mathrm{Hb}$ measurements were made every 10 min during the initial resuscitation, followed by every $2 \mathrm{~h}$ after the $\mathrm{Hb}$ goal was achieved and maintained.

\section{Measurement}

The hemodynamic parameters [HR, mean arterial pressure (MAP), SVV, CI, EVLWI], acid-base indicators [oxigen partial pressure $\left(\mathrm{PaO}_{2}\right), \mathrm{pH}$, base excess (BE), bicarbonate, lactate], plasma electrolytes (potassium, sodium, chloride), $\mathrm{Hb}$, coagulation assays [prothrombin time (PT), activated partial thromboplastin time (APTT), thrombin time (TT), fibrinogen (Fib)], and renal function [plasma urea, plasma creatinine, creatinine clearance (Clearcrea)] were checked at the following time-points: baseline, end of blood withdrawal (Shock0), before resuscitation (R0), $2 \mathrm{~h}$ after R0 (R2), $4 \mathrm{~h}$ after R0 (R4), $24 \mathrm{~h}$ after R0 (R24), and $48 \mathrm{~h}$ after R0 (R48). The LR amount, HES amount, total amount of fluid resuscitation (LR amount plus HES amount), total amount of blood withdrawn, amount of retransfused blood, urinary output, and lung wet weight: dry weight ratio were recorded at R48.

\section{Hemodynamic parameters}

HR, MAP, CI, EVLWI, and SVV were continuously measured with the PiCCO monitoring system throughout the experiment.

SVV was calculated from the mean values of four minimum and maximum SV averaged during the last $30 \mathrm{~s}$ (SVmean), as follows: SVV = (SVmax - SVmin) / SVmean.

\section{Acid-base (blood gas) parameters}

Blood samples for acid-base analysis were taken from the femoral artery and immediately analyzed in a blood gas analyzer (GEM Premier 3000; Instrumentation Laboratory, Lexington, $\mathrm{KY}$, USA) for $\mathrm{PaO}_{2}, \mathrm{pH}$, lactate, bicarbonate, and $\mathrm{BE}$. All values were adjusted for body temperature at the time of blood sampling.

\section{Electrolyte measurements}

Blood samples were taken from the CV catheter. Potassium, sodium, and chloride were determined by using a chemistry analyzer (Au5400; Olympus, Shizuoka, Japan).

\section{Renal function measurements}

Blood samples were taken from the CV catheter. Plasma urea, plasma creatinine, and 
urinary creatinine were measured by using a chemistry analyzer (Au5400; Olympus).

Clearcrea $(\mathrm{mL} / \mathrm{min})$ was assessed as an index of the glomerular filtration rate, according to the following standard formula: Clearcrea $=($ Ucrea $\times V) /$ Pcrea, where Ucrea is the concentration of creatinine in urine, $V$ is the urinary volume per minute, and Pcrea is the concentration of creatinine in plasma (Almaca et al., 2012).

\section{Coagulation measurements}

Blood samples were taken from the $\mathrm{CV}$ catheter and measured with a hemostasis analyzer (ACL TOP 700; Instrumentation Laboratory) for PT, APTT, TT, and Fib.

\section{Lung wet weight:dry weight ratio}

The animals were euthanized at $24 \mathrm{~h}$ after resuscitation, the lungs were harvested, weighed, and heated at $80^{\circ} \mathrm{C}$ in an oven for $48 \mathrm{~h}$ until the tissues were totally dry, and then reweighed. The wet weight:dry weight ratios were calculated. Data are reported as the percentage of water with the following formula (Kimberger et al., 2009): \%water $=100 \mathrm{x}$ (wet weight - dry weight) / wet weight.

\section{Statistical analysis}

All data are reported as means \pm SEM. The body weight, replacement fluid volume, total amount of blood withdrawn, amount of retransfused blood, urinary output, time to achieve the target SVV and $\mathrm{Hb}$, and lung wet weight:dry weight ratio were analyzed among groups by using one-way ANOVA. The hemodynamic, metabolic, electrolyte, renal function, and coagulation parametric data were analyzed within and among groups by using ANOVA for repeated measurements (SPSS version 11.5; SPSS Inc., Chicago, IL, USA). Statistical significance was defined as a $P$ value of $<0.05$.

\section{RESULTS}

\section{General data}

One animal in the 3:1 group did not survive until study completion. Among all study groups, there were no statistical differences with regard to body weight (Table 1).

\begin{tabular}{|c|c|c|c|c|c|c|c|}
\hline Group & $\begin{array}{l}\text { Weight } \\
(\mathrm{kg})\end{array}$ & $\begin{array}{l}\text { LR amount } \\
(\mathrm{mL})\end{array}$ & $\begin{array}{l}\text { HES amount } \\
(\mathrm{mL})\end{array}$ & $\begin{array}{l}\text { Total amount of fluid } \\
\text { resuscitation }(\mathrm{mL})\end{array}$ & $\begin{array}{c}\text { Total amount of } \\
\text { blood withdrawn }(\mathrm{mL})\end{array}$ & $\begin{array}{c}\text { Re-transfused } \\
\text { blood amount }(\mathrm{mL})\end{array}$ & $\begin{array}{c}\text { Urinary } \\
\text { output }(\mathrm{mL})\end{array}$ \\
\hline $3: 1$ & $16.3 \pm 1.2$ & $3308 \pm 770^{\dagger}$ & $1102 \pm 225^{\dagger}$ & $4412 \pm 995$ & $687 \pm 37$ & $557 \pm 97$ & $3010 \pm 939$ \\
\hline $2: 1$ & $16.1 \pm 1.6$ & $2514 \pm 677$ & $1256 \pm 209$ & $3771 \pm 886$ & $678 \pm 28$ & $532 \pm 71$ & $2603 \pm 749$ \\
\hline $1: 1$ & $15.6 \pm 1.0$ & $1502 \pm 382$ & $1501 \pm 203$ & $3004 \pm 585$ & $655 \pm 45$ & $527 \pm 69$ & $2103 \pm 575$ \\
\hline $1: 2$ & $15.8 \pm 1.1$ & $874 \pm 284^{\# \dagger \dagger}$ & $1751 \pm 339^{* 8}$ & $2625 \pm 623^{\# 8}$ & $664 \pm 39$ & $513 \pm 60$ & $1881 \pm 592$ \\
\hline $1: 3$ & $16.0 \pm 1.7$ & $653 \pm 223^{\# \$ 4}$ & $1959 \pm 365^{58 t}$ & $2611 \pm 589^{\# \S}$ & $679 \pm 32$ & $501 \pm 83$ & $1724 \pm 491^{*}$ \\
\hline
\end{tabular}

LR = lactated Ringer's solution; HES = hydroxyethyl starch; 3:1=3:1 ratio of LR: HES; 2:1=2:1 ratio of LR:HES; $1: 1=1: 1$ ratio of LR:HES; $1: 1=1: 1$ ratio of LR:HES; $1: 2=1: 2$ ratio of LR: HES; $1: 3=1: 3$ ratio of LR:HES; LR Amount, amount of infused LR; HES Amount, amount of infused HES; Total Amount of Fluid Resuscitation = amount of infused LR plus HES; ${ }^{\#} \mathrm{P}<0.05$ from Group 3:1. ${ }^{\S} \mathrm{P}<0.05$ from Group 2:1. ${ }^{\dagger} \mathrm{P}<0.05$ from Group 1:1. 
The time to achieve the target SVV and $\mathrm{Hb}$ of the 3:1, 2:1, 1:1, 1:2, and 1:3 groups was $85 \pm 23,80 \pm 29,76 \pm 14,66 \pm 18$, and $57 \pm 17 \mathrm{~min}$, respectively; the $3: 1$ group took a longer time than the $1: 3$ group $(\mathrm{P}<0.05)$. The lung wet weight: dry weight ratio of the $3: 1$, $2: 1,1: 1,1: 2$, and $1: 3$ groups was $4.2 \pm 0.3,4.0 \pm 0.4,3.9 \pm 0.3,3.8 \pm 0.3$, and $3.7 \pm 0.2$, respectively, and the 3:1 group had ratios larger than those of the 1:3 group $(\mathrm{P}<0.05)$.

\section{Fluid, blood, and urinary volume parameters}

At the end of the experiment, the 3:1 group received a greater total amount of resuscitation fluid (LR amount plus HES amount) than the 1:3 group (4412 \pm 995 vs $2611 \pm 588$ $\mathrm{mL}, \mathrm{P}<0.05)$. Thus, the $3: 1$ group received the greatest total amount of fluid resuscitation, whereas the 1:3 group received the least. Likewise, the 3:1 group received the greatest LR amount, whereas the 1:3 group received the greatest HES amount. In the 3:1 group, the greater total amount of fluid resuscitation received resulted in higher urinary outputs $(3010 \pm 939 v \mathrm{~s}$ $1724 \pm 491 \mathrm{~mL}, \mathrm{P}<0.05$ ) (Table 1) and lung wet weight:dry weight ratio than in the 3:1 group $(4.2 \pm 0.3$ vs $3.7 \pm 0.2, \mathrm{P}<0.05)$ and at baseline (Table 1, Figure 2$)$. The total amounts of fluid resuscitation and urinary outputs of the 1:2 group were larger than those of the 3:1 group (P $<0.05)$. There was no significant statistical difference in the total amount of blood withdrawn and the amount of retransfused blood in all groups.

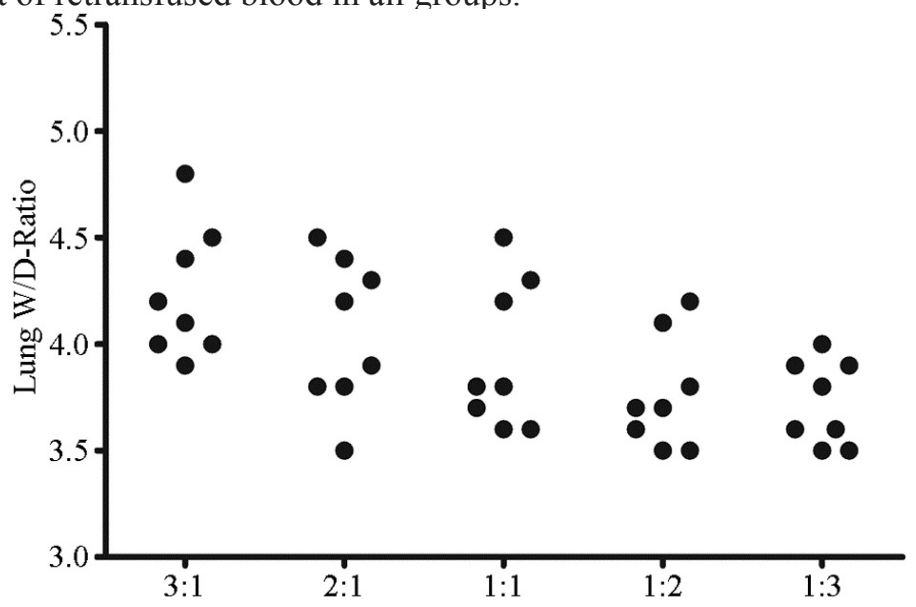

Figure 2. Lung Wet/Dry (W/R) ratio. $3: 1=3: 1$ ratio of LR:HES; $2: 1=2: 1$ ratio of LR:HES; $1: 1=1: 1$ ratio of LR:HES; $1: 2=1: 2$ ratio of LR: HES; 1:3 = 1:3 ratio of LR: HES; ${ }^{*} \mathrm{P}<0.05$ from baseline. ${ }^{~} \mathrm{P}<0.05$ from Group $3: 1$.

\section{Hemodynamic, electrolyte, acid-base, renal function, and coagulation parameters}

During the period from Shock0 to R0, the proposed model of hemorrhagic shock produced a significant decrease in MAP and CI, whereas SVV and HR increased compared with the baseline values $(\mathrm{P}<0.05)$ (Table 2). After resuscitation with fluids and shed blood, the HR, MAP, SVV, and CI gradually returned to the baseline levels at R4. However, the HR, MAP, SVV, and CI did not show a statistically significant change in all groups at all time points. The EVLWI of the 3:1 group was higher than that of the 3:1 group and at baseline (Table 2, Figure 3). 
Table 2. Hemodynamic, acid-base, electrolytes, and renal function parameters.

\begin{tabular}{|c|c|c|c|c|c|c|c|}
\hline Parameters & Group & Baseline & Shock0 & R0 & $\mathrm{R} 2$ & $\mathrm{R} 4$ & $\mathrm{R} 24$ \\
\hline \multirow[t]{5}{*}{ HR (bpm) } & $3: 1$ & $145 \pm 17$ & $167 \pm 18^{*}$ & $182 \pm 21^{*}$ & $160 \pm 17$ & $153 \pm 18$ & $151 \pm 18$ \\
\hline & $2: 1$ & $146 \pm 21$ & $161 \pm 21^{*}$ & $168 \pm 20 *$ & $154 \pm 23$ & $156 \pm 16$ & $147 \pm 20$ \\
\hline & $1: 1$ & $138 \pm 14$ & $151 \pm 15^{*}$ & $170 \pm 17 *$ & $142 \pm 24$ & $144 \pm 21$ & $143 \pm 15$ \\
\hline & $1: 2$ & $144 \pm 16$ & $166 \pm 14^{*}$ & $167 \pm 24 *$ & $150 \pm 20$ & $152 \pm 13$ & $141 \pm 13$ \\
\hline & $1: 3$ & $143 \pm 18$ & $156 \pm 15^{*}$ & $179 \pm 20^{*}$ & $156 \pm 15$ & $140 \pm 17$ & $138 \pm 14$ \\
\hline \multirow[t]{5}{*}{ MAP (mmHg) } & $3: 1$ & $126 \pm 15$ & $46 \pm 6^{*}$ & $74 \pm 5^{*}$ & $131 \pm 15$ & $134 \pm 13$ & $139 \pm 16$ \\
\hline & $2: 1$ & $120 \pm 17$ & $43 \pm 7^{*}$ & $71 \pm 4 *$ & $127 \pm 13$ & $132 \pm 16$ & $131 \pm 17$ \\
\hline & $1: 1$ & $123 \pm 18$ & $47 \pm 5^{*}$ & $69 \pm 6^{*}$ & $111 \pm 17$ & $124 \pm 17$ & $132 \pm 18$ \\
\hline & $1: 2$ & $114 \pm 17$ & $40 \pm 4^{*}$ & $72 \pm 6^{*}$ & $122 \pm 9$ & $125 \pm 12$ & $128 \pm 11$ \\
\hline & $1: 3$ & $117 \pm 16$ & $44 \pm 5^{*}$ & $73 \pm 5^{*}$ & $117 \pm 10$ & $129 \pm 13$ & $127 \pm 13$ \\
\hline \multirow[t]{5}{*}{ SVV (\%) } & $3: 1$ & $9.3 \pm 1.6$ & $23.7 \pm 3.2 *$ & $28.8 \pm 4.6^{*}$ & $8.9 \pm 1.7$ & $9.3 \pm 1.2$ & $9.4 \pm 0.9$ \\
\hline & $2: 1$ & $9.6 \pm 1.8$ & $25.0 \pm 3.8^{*}$ & $27.2 \pm 3.3^{*}$ & $9.2 \pm 1.1$ & $9.5 \pm 1.4$ & $9.8 \pm 1.1$ \\
\hline & $1: 1$ & $9.5 \pm 1.4$ & $24.8 \pm 2.1^{*}$ & $26.7 \pm 2.7^{*}$ & $9.8 \pm 1.4$ & $9.8 \pm 1.1$ & $9.3 \pm 1.3$ \\
\hline & $1: 2$ & $9.4 \pm 1.6$ & $22.5 \pm 3.6^{*}$ & $28.6 \pm 3.5^{*}$ & $9.2 \pm 1.8$ & $9.3 \pm 0.8$ & $9.6 \pm 1.2$ \\
\hline & $1: 3$ & $9.8 \pm 1.7$ & $23.8 \pm 3.1^{*}$ & $27.4 \pm 2.9^{*}$ & $9.5 \pm 0.9$ & $9.2 \pm 1.0$ & $9.3 \pm 1.0$ \\
\hline \multirow[t]{5}{*}{$\mathrm{CI}\left(\mathrm{L} \cdot \mathrm{min}^{-1} \cdot \mathrm{m}^{-2}\right)$} & $3: 1$ & $3.8 \pm 0.2$ & $1.9 \pm 0.2 *$ & $1.5 \pm 0.3 *$ & $4.4 \pm 0.2$ & $4.2 \pm 0.7$ & $5.4 \pm 0.6^{*}$ \\
\hline & $2: 1$ & $3.4 \pm 0.3$ & $1.8 \pm 0.3^{*}$ & $1.4 \pm 0.2^{*}$ & $3.4 \pm 0.3$ & $4.1 \pm 0.5$ & $5.0 \pm 0.9 *$ \\
\hline & $1: 1$ & $3.5 \pm 0.6$ & $1.6 \pm 0.5^{*}$ & $1.5 \pm 0.3^{*}$ & $3.9 \pm 0.4$ & $4.0 \pm 0.4$ & $5.1 \pm 0.4 *$ \\
\hline & $1: 2$ & $3.6 \pm 0.4$ & $1.7 \pm 0.2 *$ & $1.6 \pm 0.1^{*}$ & $3.8 \pm 0.2$ & $3.8 \pm 0.3$ & $4.9 \pm 0.3^{*}$ \\
\hline & $1: 3$ & $3.7 \pm 0.5$ & $1.8 \pm 0.4^{*}$ & $1.7 \pm 0.2^{*}$ & $3.4 \pm 0.3$ & $3.9 \pm 0.6$ & $5.2 \pm 0.8^{*}$ \\
\hline \multirow[t]{5}{*}{ EVLWI (mL/kg) } & $3: 1$ & $9.7 \pm 0.6$ & $9.2 \pm 0.8$ & $9.4 \pm 0.9$ & $10.2 \pm 0.8$ & $10.6 \pm 0.2$ & $11.4 \pm 0.4 *$ \\
\hline & $2: 1$ & $10.3 \pm 0.2$ & $9.7 \pm 0.9$ & $9.9 \pm 0.4$ & $10.0 \pm 1.0$ & $10.5 \pm 0.3$ & $10.7 \pm 0.6$ \\
\hline & $1: 1$ & $9.6 \pm 0.5$ & $9.5 \pm 0.4$ & $9.7 \pm 0.8$ & $9.8 \pm 1.1$ & $10.3 \pm 0.5$ & $10.5 \pm 0.3$ \\
\hline & $1: 2$ & $10.2 \pm 0.4$ & $9.9 \pm 0.5$ & $9.5 \pm 0.6$ & $9.9 \pm 0.8$ & $10.4 \pm 0.4$ & $10.3 \pm 0.5$ \\
\hline & $1: 3$ & $9.9 \pm 0.3$ & $9.4 \pm 0.7$ & $9.6 \pm 0.8$ & $9.7 \pm 0.9$ & $9.9 \pm 0.6$ & $10.1 \pm 0.3^{\#}$ \\
\hline \multirow[t]{5}{*}{ Hemoglobin $(\mathrm{g} / \mathrm{dL})$} & $3: 1$ & $14.2 \pm 1.4$ & $13.9 \pm 1.1$ & $15.2 \pm 1.3$ & $10.7 \pm 1.2$ & $10.6 \pm 1.0$ & $10.4 \pm 0.6$ \\
\hline & $2: 1$ & $13.8 \pm 0.6$ & $13.2 \pm 0.8$ & $14.8 \pm 1.5$ & $11.0 \pm 1.3$ & $10.7 \pm 0.6$ & $10.3 \pm 0.3$ \\
\hline & $1: 1$ & $13.6 \pm 1.2$ & $13.4 \pm 0.6$ & $14.3 \pm 1.9$ & $10.6 \pm 1.2$ & $10.5 \pm 0.5$ & $10.4 \pm 0.6$ \\
\hline & $1: 2$ & $13.3 \pm 0.7$ & $13.5 \pm 0.8$ & $14.8 \pm 2.4$ & $10.7 \pm 0.7$ & $10.6 \pm 0.7$ & $10.5 \pm 0.5$ \\
\hline & $1: 3$ & $13.4 \pm 0.9$ & $13.6 \pm 0.9$ & $14.3 \pm 1.2$ & $10.9 \pm 0.8$ & $10.7 \pm 0.9$ & $10.3 \pm 0.4$ \\
\hline \multirow[t]{5}{*}{$\mathrm{pH}$} & $3: 1$ & $7.39 \pm 0.03$ & $7.34 \pm 0.06$ & $7.20 \pm 0.04 *$ & $7.30 \pm 0.03$ & $7.35 \pm 0.05$ & $7.39 \pm 0.03$ \\
\hline & $2: 1$ & $7.40 \pm 0.06$ & $7.35 \pm 0.05$ & $7.17 \pm 0.05^{*}$ & $7.32 \pm 0.06$ & $7.36 \pm 0.07$ & $7.37 \pm 0.04$ \\
\hline & $1: 1$ & $7.36 \pm 0.05$ & $7.32 \pm 0.04$ & $7.21 \pm 0.07 *$ & $7.33 \pm 0.05$ & $7.37 \pm 0.05$ & $7.35 \pm 0.05$ \\
\hline & $1: 2$ & $7.38 \pm 0.04$ & $7.33 \pm 0.07$ & $7.19 \pm 0.06^{*}$ & $7.32 \pm 0.04$ & $7.35 \pm 0.04$ & $7.32 \pm 0.04$ *\# \\
\hline & $1: 3$ & $7.37 \pm 0.04$ & $7.32 \pm 0.05$ & $7.18 \pm 0.05^{*}$ & $7.34 \pm 0.07$ & $7.38 \pm 0.06$ & $7.31 \pm 0.03$ *\# \\
\hline \multirow[t]{5}{*}{$\mathrm{BE}$} & $3: 1$ & $-2.6 \pm 0.8$ & $-3.3 \pm 0.7$ & $-8.9 \pm 1.1 *$ & $-3.1 \pm 0.4$ & $-3.2 \pm 0.7$ & $-2.1 \pm 0.3$ \\
\hline & $2: 1$ & $-2.2 \pm 0.5$ & $-2.5 \pm 0.4$ & $-9.7 \pm 1.3 *$ & $-3.5 \pm 0.3$ & $-2.8 \pm 0.6$ & $-2.8 \pm 0.7$ \\
\hline & $1: 1$ & $-2.8 \pm 0.9$ & $-3.2 \pm 0.8$ & $-8.7 \pm 0.8 *$ & $-3.4 \pm 0.5$ & $-2.3 \pm 0.4$ & $-2.9 \pm 0.4$ \\
\hline & $1: 2$ & $-2.5 \pm 0.6$ & $-2.5 \pm 0.7$ & $9.5 \pm 1.2 *$ & $-2.8 \pm 0.4$ & $-2.1 \pm 0.3$ & $-3.7 \pm 0.5^{\# *}$ \\
\hline & $1: 3$ & $-2.9 \pm 0.7$ & $-3.1 \pm 0.6$ & $-9.8 \pm 0.9^{*}$ & $-3.2 \pm 0.6$ & $-2.2 \pm 0.5$ & $-4.9 \pm 0.4^{\# *}$ \\
\hline \multirow[t]{5}{*}{ Lactate (mM) } & $3: 1$ & $2.1 \pm 0.7$ & $2.5 \pm 0.6$ & $4.7 \pm 0.6^{*}$ & $3.1 \pm 0.9$ & $3.0 \pm 0.7$ & $1.6 \pm 0.6$ \\
\hline & $2: 1$ & $1.6 \pm 0.4$ & $1.9 \pm 0.4$ & $4.4 \pm 0.7^{*}$ & $3.4 \pm 0.5$ & $2.8 \pm 0.5$ & $1.3 \pm 0.4$ \\
\hline & $1: 1$ & $1.8 \pm 0.6$ & $2.1 \pm 0.2$ & $4.6 \pm 0.5^{*}$ & $3.0 \pm 0.3$ & $2.6 \pm 0.2$ & $1.7 \pm 0.3$ \\
\hline & $1: 2$ & $1.2 \pm 0.5$ & $1.7 \pm 0.8$ & $4.8 \pm 0.4^{*}$ & $2.7 \pm 0.6$ & $2.3 \pm 0.4$ & $1.5 \pm 0.7$ \\
\hline & $1: 3$ & $2.0 \pm 0.6$ & $2.3 \pm 0.4$ & $4.9 \pm 0.8^{*}$ & $2.5 \pm 0.4$ & $2.1 \pm 0.8$ & $1.4 \pm 0.5$ \\
\hline \multirow[t]{5}{*}{ Bicarbonate (mM) } & $3: 1$ & $23.3 \pm 3.8$ & $20.8 \pm 2.5$ & $15.3 \pm 1.7^{*}$ & $20.1 \pm 1.5$ & $22.6 \pm 2.1$ & $24.4 \pm 3.6$ \\
\hline & $2: 1$ & $22.7 \pm 2.6$ & $21.2 \pm 2.1$ & $14.6 \pm 2.5^{*}$ & $20.3 \pm 1.2$ & $22.3 \pm 1.2$ & $23.8 \pm 3.3$ \\
\hline & $1: 1$ & $21.3 \pm 2.1$ & $20.4 \pm 3.7$ & $15.2 \pm 4.2^{*}$ & $21.5 \pm 1.0$ & $23.4 \pm 1.3$ & $24.5 \pm 2.0$ \\
\hline & $1: 2$ & $21.1 \pm 2.4$ & $20.5 \pm 2.2$ & $15.0 \pm 2.4^{*}$ & $20.2 \pm 1.4$ & $23.2 \pm 1.5$ & $24.3 \pm 3.5$ \\
\hline & $1: 3$ & $22.0 \pm 3.9$ & $20.1 \pm 4.3$ & $14.8 \pm 2.1^{*}$ & $21.8 \pm 1.3$ & $24.1 \pm 1.1$ & $23.7 \pm 3.2$ \\
\hline \multirow{5}{*}{$\mathrm{PaO}_{2}(\mathrm{mmHg})$} & $3: 1$ & $148 \pm 16$ & $136 \pm 10$ & $137 \pm 14$ & $132 \pm 16$ & $124 \pm 17$ & $110 \pm 8^{*}$ \\
\hline & $2: 1$ & $139 \pm 19$ & $133 \pm 13$ & $126 \pm 13$ & $130 \pm 12$ & $128 \pm 7$ & $128 \pm 17$ \\
\hline & $1: 1$ & $141 \pm 12$ & $129 \pm 7$ & $129 \pm 10$ & $136 \pm 13$ & $134 \pm 9$ & $130 \pm 14$ \\
\hline & $1: 2$ & $144 \pm 17$ & $131 \pm 12$ & $132 \pm 15$ & $138 \pm 18$ & $135 \pm 16$ & $133 \pm 9$ \\
\hline & $1: 3$ & $146 \pm 11$ & $135 \pm 8$ & $127 \pm 9$ & $129 \pm 11$ & $137 \pm 12$ & $138 \pm 11^{\#}$ \\
\hline \multirow[t]{5}{*}{$\mathrm{K}^{+}(\mathrm{mM})$} & $3: 1$ & $4.8 \pm 1.2$ & $4.5 \pm 0.8$ & $4.2 \pm 0.7$ & $4.3 \pm 0.5$ & $3.9 \pm 0.9$ & $3.0 \pm 0.8^{*}$ \\
\hline & $2: 1$ & $4.7 \pm 0.9$ & $4.3 \pm 0.4$ & $4.2 \pm 0.6$ & $4.1 \pm 0.8$ & $3.7 \pm 0.5$ & $3.1 \pm 0.7^{*}$ \\
\hline & $1: 1$ & $4.4 \pm 1.4$ & $4.2 \pm 0.5$ & $4.1 \pm 0.3$ & $4.2 \pm 0.4$ & $3.5 \pm 0.5$ & $3.2 \pm 0.5^{*}$ \\
\hline & $1: 2$ & $4.3 \pm 0.8$ & $4.0 \pm 0.7$ & $3.8 \pm 0.5$ & $3.9 \pm 0.5$ & $3.6 \pm 0.8$ & $3.0 \pm 0.6^{*}$ \\
\hline & $1: 3$ & $4.6 \pm 1.0$ & $4.3 \pm 0.6$ & $4.2 \pm 0.4$ & $4.1 \pm 0.9$ & $3.7 \pm 1.2$ & $2.8 \pm 0.7^{*}$ \\
\hline \multirow{2}{*}{$\mathrm{Na}^{+}(\mathrm{mM})$} & $3: 1$ & $140 \pm 8$ & $145 \pm 8$ & $140 \pm 5$ & $139 \pm 6$ & $143 \pm 8$ & $139 \pm 4$ \\
\hline & $2: 1$ & $137 \pm 7$ & $140 \pm 7$ & $137 \pm 6$ & $140 \pm 7$ & $145 \pm 4$ & $142 \pm 6$ \\
\hline
\end{tabular}

Continued on next page 


\begin{tabular}{|c|c|c|c|c|c|c|c|}
\hline Parameters & Group & Baseline & Shock0 & R0 & $\mathrm{R} 2$ & $\mathrm{R} 4$ & $\mathrm{R} 24$ \\
\hline & $1: 1$ & $145 \pm 6$ & $139 \pm 6$ & $145 \pm 7$ & $142 \pm 5$ & $142 \pm 5$ & $144 \pm 4$ \\
\hline & $1: 2$ & $140 \pm 5$ & $143 \pm 9$ & $142 \pm 6$ & $145 \pm 3$ & $145 \pm 8$ & $149 \pm 5^{* \#}$ \\
\hline & $1: 3$ & $139 \pm 4$ & $142 \pm 5$ & $139 \pm 8$ & $144 \pm 4$ & $148 \pm 7$ & $151 \pm 6^{* \#}$ \\
\hline \multirow[t]{5}{*}{$\mathrm{Cl}^{-}(\mathrm{mM})$} & $3: 1$ & $103 \pm 4$ & $100 \pm 8$ & $105 \pm 6$ & $102 \pm 5$ & $101 \pm 9$ & $99 \pm 5$ \\
\hline & $2: 1$ & $106 \pm 7$ & $108 \pm 7$ & $103 \pm 5$ & $101 \pm 7$ & $105 \pm 8$ & $106 \pm 6$ \\
\hline & $1: 1$ & $104 \pm 6$ & $102 \pm 3$ & $106 \pm 9$ & $105 \pm 6$ & $104 \pm 5$ & $109 \pm 4$ \\
\hline & $1: 2$ & $100 \pm 7$ & $103 \pm 6$ & $104 \pm 8$ & $106 \pm 8$ & $108 \pm 7$ & $112 \pm 5^{* \#}$ \\
\hline & $1: 3$ & $102 \pm 8$ & $106 \pm 5$ & $103 \pm 10$ & $108 \pm 3$ & $111 \pm 6$ & $114 \pm 7 * \#$ \\
\hline \multirow[t]{5}{*}{ Clearcrea $(\mathrm{mL} / \mathrm{min})$} & $3: 1$ & $62 \pm 7$ & $44 \pm 10 *$ & $14 \pm 5^{*}$ & $57 \pm 10$ & $60 \pm 7$ & $63 \pm 9$ \\
\hline & $2: 1$ & $64 \pm 6$ & $37 \pm 8^{*}$ & $17 \pm 13^{*}$ & $58 \pm 7$ & $59 \pm 5$ & $60 \pm 13$ \\
\hline & $1: 1$ & $63 \pm 9$ & $39 \pm 12 *$ & $13 \pm 8^{*}$ & $57 \pm 9$ & $58 \pm 9$ & $61 \pm 7$ \\
\hline & $1: 2$ & $66 \pm 9$ & $45 \pm 7^{*}$ & $15 \pm 9 *$ & $55 \pm 7$ & $57 \pm 11$ & $54 \pm 6^{* \#}$ \\
\hline & $1: 3$ & $63 \pm 8$ & $41 \pm 9^{*}$ & $12 \pm 6^{*}$ & $54 \pm 6$ & $55 \pm 9$ & $52 \pm 8$ *\# \\
\hline
\end{tabular}

$\mathrm{HR}=$ heart rate; $\mathrm{MAP}=$ mean arterial pressure; $\mathrm{SVV}=$ stroke volume variation; $\mathrm{CI}=$ cardiac index; EVLWI $=$ extravascular lung water index; $\mathrm{BE}=$ base excess; $\mathrm{PaO}_{2}=$ partial pressure of oxygen; $\mathrm{K}^{+}=$plasma potassium concentration; $\mathrm{Na}^{+}=$plasma sodium concentration; $\mathrm{Cl}^{-} \stackrel{2}{=}$ plasma chloride concentration; Clearcrea = creatinine clearance; 3:1=3:1 ratio of LR:HES; 2:1=2:1 ratio of LR: HES; $1: 1=1: 1$ ratio of LR: HES; $1: 2=1: 2$ ratio of LR:HES; HES; 1:3=1:3 ratio of LR: HES; ${ }^{\mathrm{P}}<0.05$ from baseline. \#P $<0.05$ from Group 3:1.

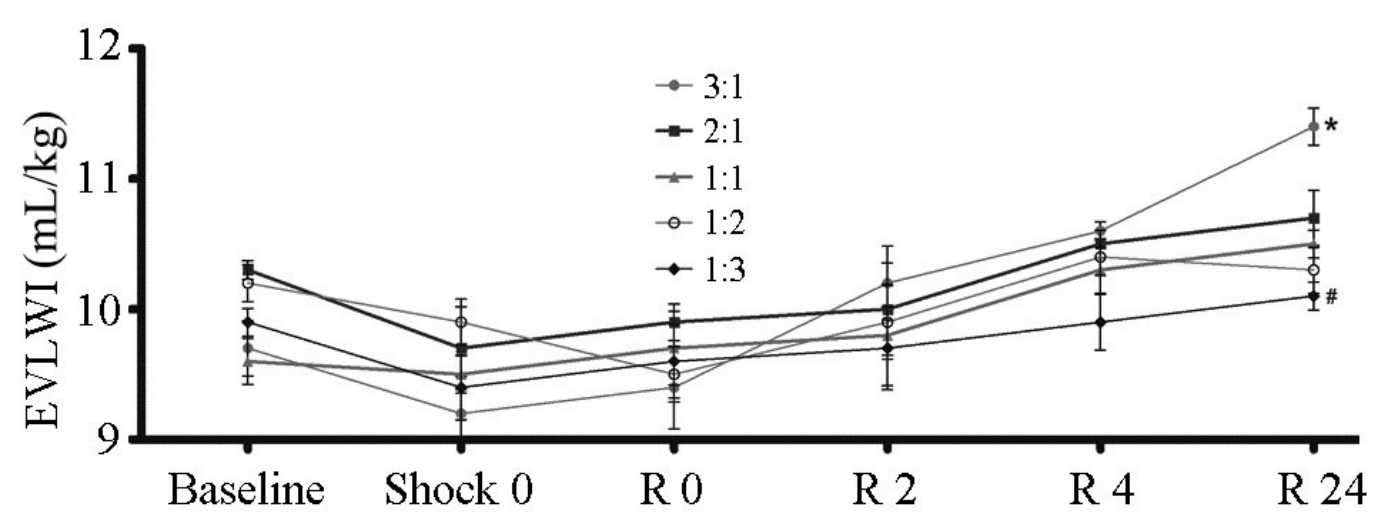

Figure 3. Extravascular lung water index (EVLWI). ${ }^{*} \mathrm{P}<0.05$ from baseline. ${ }^{*} \mathrm{P}<0.05$ from Group 3:1.

During the period from Shock0 to R0, hemorrhagic shock led to declines in $\mathrm{pH}, \mathrm{BE}$, and bicarbonate, and increase in lactate compared with the baseline values $(\mathrm{P}<0.05)$, reaching the nadir and peak at R0. After resuscitation with fluids and shed blood, the $\mathrm{pH}, \mathrm{BE}$, and bicarbonate gradually increased and approached the baseline values at $\mathrm{R} 4$ (Table 2). The $\mathrm{PaO}_{2}$ values in the 3:1 group was lower than those in the 3:1 group and the baseline value at R24 $(\mathrm{P}<0.05)$ (Table 2, Figure 4). After the infusion of larger amounts of HES containing $0.9 \%$ sodium chloride, the plasma chloride (Figure 5) and sodium levels of the 1:3 and 1:2 groups increased statistically significantly at R24 compared with the 3:1 group and at baseline ( $<<$ 0.05 ) (Table 2). Similarly, the plasma $\mathrm{pH}$ and $\mathrm{BE}$ in the 1:3 and 1:2 groups at R24 were significantly decreased compared with the $3: 1$ group and at baseline $(\mathrm{P}<0.05)$ (Table 2). At R24, there were no significant differences in plasma $\mathrm{pH}$ and $\mathrm{BE}$ in the $2: 1$ and 1:1 groups compared with the baseline values $(\mathrm{P}>0.05)$. The plasma potassium of all groups at $\mathrm{R} 24$ was significantly lower than the baseline values $(\mathrm{P}<0.05)$ (Table 2$)$. 


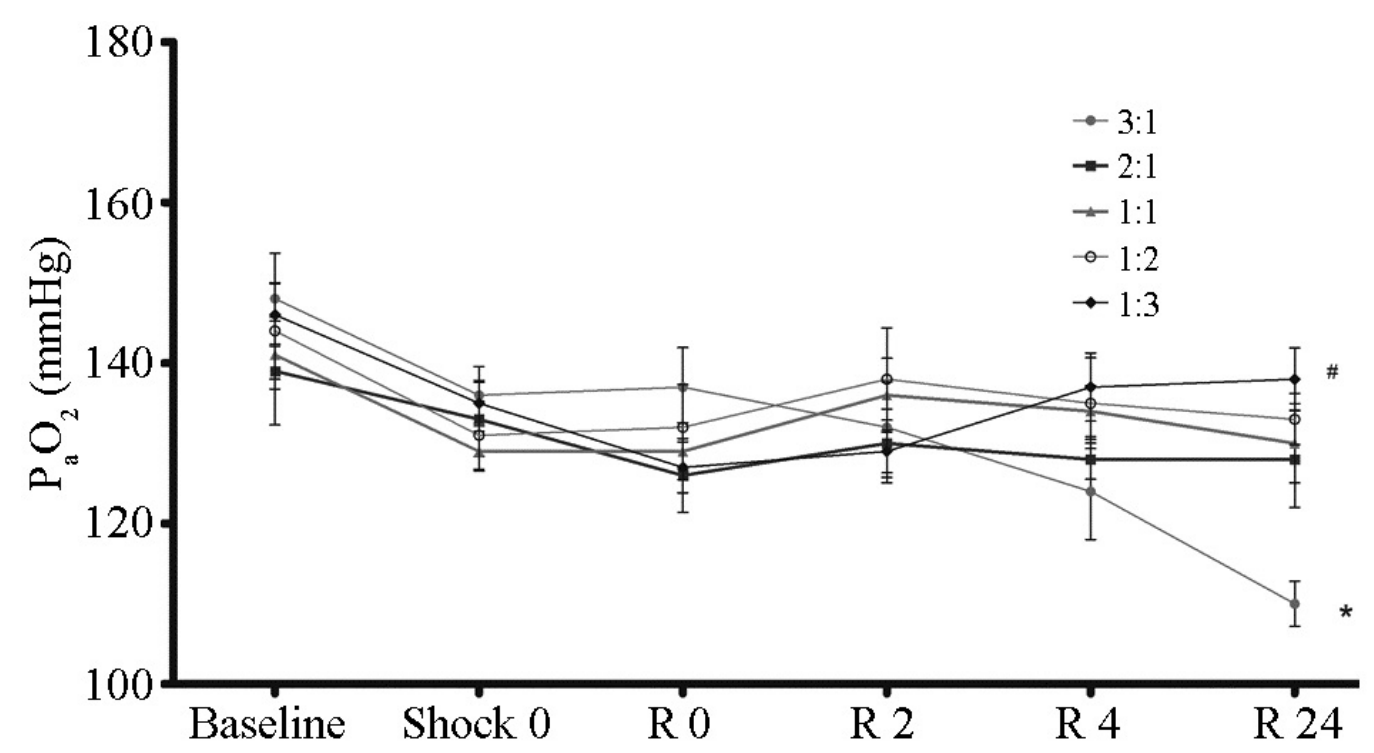

Figure 4. Partial pressure of oxygen $\left(\mathrm{PaO}_{2}\right)$. ${ }^{*} \mathrm{P}<0.05$ from baseline. ${ }^{*} \mathrm{P}<0.05$ from Group 3:1.

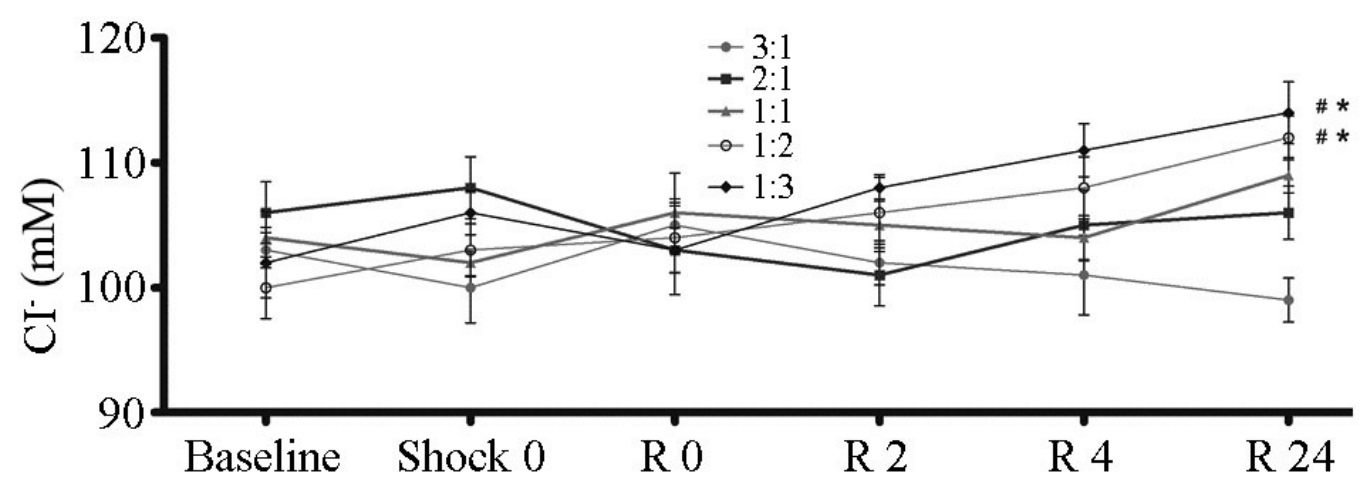

Figure 5. Plasma chloride concentration $\left(\mathrm{Cl}^{-}\right) .{ }^{*} \mathrm{P}<0.05$ from baseline. ${ }^{*} \mathrm{P}<0.05$ from Group 3:1.

There was no significant statistical difference in plasma urea and plasma creatinine in all groups at all time points (not shown). The baseline values of Clearcrea measured in each group were found to be similar $(\mathrm{P}>0.05)$. During hemorrhagic shock (from Shock0 to R0), the Clearcrea of all groups decreased dramatically and reached their nadirs at R0. After resuscitation with fluids and shed blood, the Clearcrea gradually increased and approached baseline levels at R4, with the 3:1 group having the lowest Clearcrea at R24 among all groups. The Clearcrea values of the 1:3 and 1:2 groups at R24 were lower than that of the $3: 1$ group and at baseline $(\mathrm{P}<0.05)$ (Table 2, Figure 6). At R24, there were no significant difference in Clearcrea in the 2:1 and 1:1 groups compared with the baseline ( $\mathrm{P}>0.05)$ (Table 2, Figure 6). 
There was no significant statistical difference in coagulation parameters (PT, APTT, $\mathrm{TT}$, and Fib) in all groups at all time points (not shown).

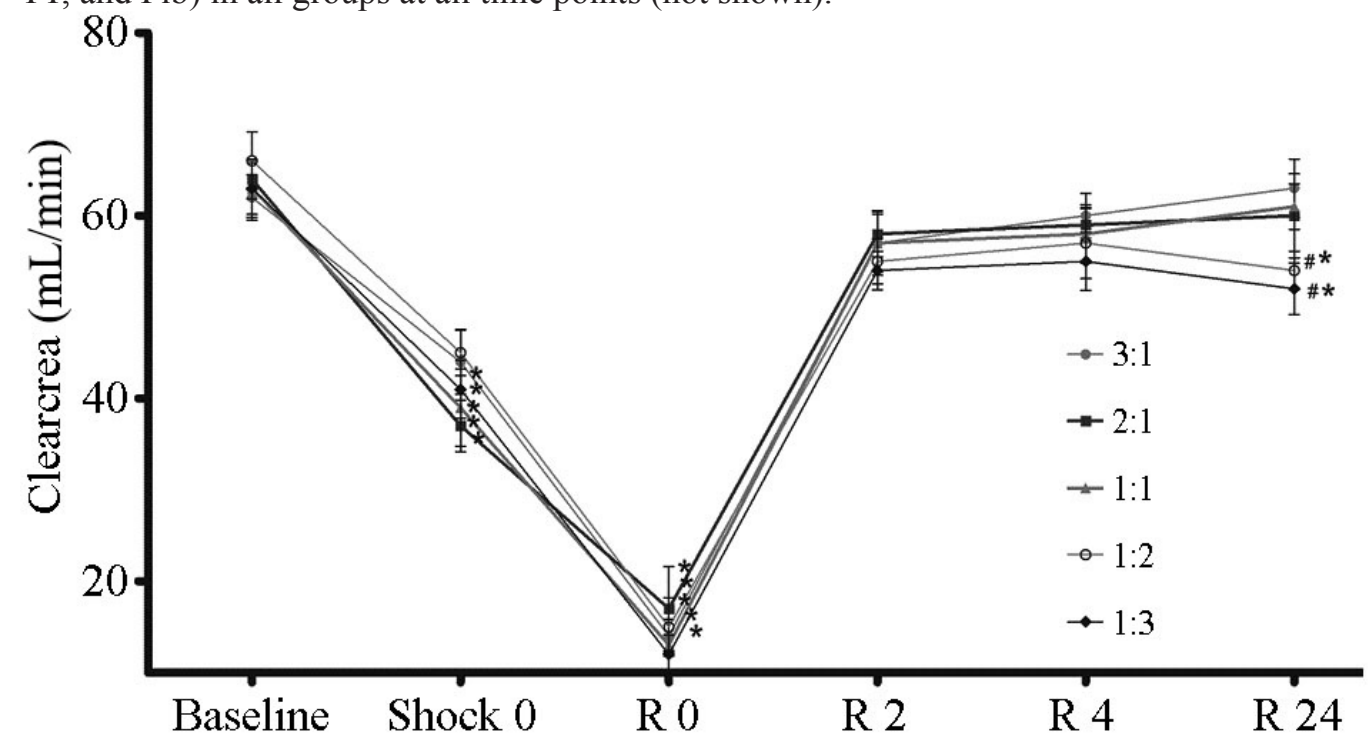

Figure 6. Creatinine clearance (Clearcrea). ${ }^{*} \mathrm{P}<0.05$ from baseline. ${ }^{*} \mathrm{P}<0.05$ from Group 3:1.

\section{DISCUSSION}

In this study, we investigated the effects of goal-directed fluid therapy with different LR: HES ratios on the acid-base and electrolyte balances, EVLW, and renal and coagulation functions in a canine hemorrhagic shock model. We tested the hypothesis that resuscitation with fluid of different LR: HES ratios would probably result in different effects on acid-base, and electrolyte balances, EVLWI, and renal function.

EVLWI is defined as the extravascular fluid in the lung at any moment, including the intracellular fluid of inflammatory, endothelial, and epithelial cells in the extravascular space, as well as alveolar and interstitial fluid and intrapulmonary lymph (Phillips at al., 2009). The amount of EVLWI represents a dynamic balance between factors that cause fluid to accumulate in the lungs and those that carry fluid out of the lungs.

In this study, to achieve maximal CO and stroke volume, the group with an LR: HES ratio of 3:1 received the largest LR amounts and total fluid resuscitation amounts. Because crystalloids (e.g., LR) are poor plasma expanders, $80 \%$ of the given volume would have shifted into the extracellular space (Almaca et al., 2012). The shift from the intravascular space can be aggravated by endothelial injury, capillary leakage, and accumulating extracellular edema, all of which are common pathophysiological mechanisms during hemorrhagic shock (Ertmer et al., 2011)

In our 3:1 group, we observed pulmonary interstitial fluid accumulation, which led to an increase in the EVLWI and lung wet weight:dry weight ratios, and consequently a decrease in $\mathrm{PaO}_{2}$ compared with the 1:3 group and the baseline value. In contrast to crystalloid solutions, colloid solutions (e.g., HES) theoretically provide longer and prolonged resuscitation 
owing to their extended intravascular longevity or circulatory lifespan (Cabrales et al., 2007).

Thus, as expected, the 1:3 group received smaller total fluid resuscitation amounts that resulted in lower EVLWI, wet weight:dry weight ratios, and $\mathrm{PaO}_{2}$ indices than those in the 3:1 group at R24. Therefore, using crystalloid for goal-directed fluid therapy resulted in greater resuscitation volumes than using colloid. Lang et al. (2001) demonstrated this result by studying the effects of $6 \%$ HES and LR on tissue oxygen tension in patients undergoing major abdominal surgery, in whom colloids may reduce endothelial tissue edema, and crystalloids may cause endothelial tissue swelling and increase the oxygen diffusion distance.

In this experiment, $6 \%$ HES $130 / 0.4, \mathrm{pH} 4.0$ to 5.5 [osmolality $308 \mathrm{mOsM}$; electrolytes (M): $\mathrm{Na}^{+} 154, \mathrm{Cl}^{-} 154$ ], and LR, pH 6.5 [osmolality $272 \mathrm{mOsM}$; electrolytes (M): $\mathrm{Na}^{+}$ $130, \mathrm{Cl}^{-} 109, \mathrm{~K}^{+} 4, \mathrm{Ca}^{2+}$ 2.7, lactate 27.7$]$, were used as resuscitation fluids. In essence, $6 \%$ HES 130/0.4 is a kind of saline-based colloid solution, whereas LR is a balanced crystalloid solution. Although saline-based solutions have been associated with disturbed acid-base balance because of their non-physiological electrolyte composition and $\mathrm{pH}$ (Wilcox, 1983; Scheingraber et al., 1999), they remain to be popular choices for volume replacement therapy in perioperative care (Almaca et al., 2012). In addition, saline-based solutions are known to be more frequently associated with hyperchloremic acidosis, owing to their high levels of chloride, resulting in renal vascular constriction and potentially to renal dysfunction (Williams et al., 1999; Brill et al., 2002; Almaca et al., 2012).

In our model, the plasma chloride values in the 1:3 group $(114 \pm 7 \mathrm{M})$ and 1:2 group $(112 \pm 5 \mathrm{M})$ were higher than that in the $3: 1$ group $(99 \pm 5 \mathrm{M})$ and at baseline, being infused with a greater amount of HES at R24. This may be because HES has a chloride content of 154 $\mathrm{M}$, whereas LR has a chloride content of $109 \mathrm{M}$. In addition, our study showed that $\mathrm{BE}$ and $\mathrm{pH}$ were significantly lower in the 1:3 and 1:2 groups (saline-based colloid solution infused) at R4 compared with the 3:1 group (greater amount of balanced crystalloid solution infused). However, no difference was found with respect to lactate levels. This suggests that resuscitation with large amounts of HES leads to hyperchloremia, which produces a relative hyperchloremic acidosis. Furthermore, hyperchloremia may lead to renal function decrease by triggering renal vascular constriction and decreased renal perfusion (Almaca et al., 2012). Conversely, buffers (i.e., lactate) found in LR may be converted to bicarbonate in the liver and contribute to a lesser degree of acidemia, or increase the $\mathrm{pH}$ (Haisch et al., 2001).

Almaca et al. (2012) used acetate-balanced colloid and crystalloid for the evaluation of renal oxygenation in a murine hemorrhagic shock model. They found that resuscitation with $0.9 \% \mathrm{NaCl}$ and acetated Ringer's solution and HES dissolved in saline led to hyperchloremic acidosis and progressive renal vasoconstriction, whereas resuscitation with the HES dissolved in RA did not. However, Feldheiser et al. (2013) found no renal impairment secondary to colloid fluid administration when targeted at optimizing cardiac preload in 50 patients with primary ovarian cancer undergoing cytoreductive surgery. In our experiment, the plasma $\mathrm{pH}$ and $\mathrm{BE}$ in the 1:3 and 1:2 groups at R24 were significantly decreased compared with the 3:1 group and at baseline $(\mathrm{P}<0.05)$. Meanwhile, greater HES administration resulted in changes in Clearcrea in the 1:3 and 1:2 groups at R24, which were lower than those of the 3:1 group and at baseline (P $<0.05)$. At R24, there were no significant differences in plasma $\mathrm{pH}, \mathrm{BE}$, and Clearcrea in 2:1 and 1:1 groups compared with the baseline $(\mathrm{P}>0.05)$. This suggests that resuscitation with large amounts of HES (saline-based colloid solution) may have induced hyperchloremia acidosis and renal function impairment as a consequence of acute hemorrhagic shock.

In our model, the plasma sodium values in the 1:3 and 1:2 groups at R24 were higher 
than those in the 3:1 group and at baseline, with the 1:3 and 1:2 groups having received more HES at R24. This suggests that resuscitation with large amounts of HES containing $0.9 \%$ $\mathrm{NaCl}$ would result in hypernatremia. It was undoubtedly caused by the perfusion of HES with a higher sodium concentration $(154 \mathrm{M})$ than LR $(130 \mathrm{M})$. Usually, the renal tubules adjust and maintain the stable plasma sodium concentration through $\mathrm{H}^{+}-\mathrm{Na}^{+}$and $\mathrm{Na}^{+}-\mathrm{K}^{+}$changes in the normal condition. However, the renal tubules are prone to be affected and impaired in the presence of hemorrhagic shock. Furthermore, because of the relatively larger molecule of HES, the clearance of HES was delayed in the presence of hemorrhagic shock. The study of Walsh et al. (2008) also demonstrated our outcomes. They found that patients routinely received significantly greater amounts of sodium and fluid were physiologically impaired, in a prospective audit of postoperative fluid management practices in 106 consecutive patients undergoing laparotomy in a UK general surgical unit over a 6-month period in 2003.

With respect to effects on coagulation function, several studies have documented an increased bleeding tendency induced by the administration of HES, especially high-molecular HES (Fries et al., 2002; Mauch et al., 2012). HES solutions cause a syndrome similar to the von Willebrand type I syndrome, which is characterized by a diminished factor VIII activity, decreased von Willebrand factor plasma levels, and a decreased factor VIII-associated ristocetin cofactor in plasma. Besides the quantity of the administered volume, the effect of HES on the coagulation system depends on the concentration, molecular weight, degree of substitution, and on the ratio (C1:C4) of substitution (Warren and Durieux, 1997 ).

In our experiment, we observed no significant intergroup differences in coagulation parameters (PT, APTT, TT, and Fib) throughout the entire protocol, even when all of the groups received amounts exceeding the maximal recommended dose of $0.6 \%$ HES 130/0.4 (50 mL/ $\mathrm{kg}$ ). Our results are corroborated by the study of Yoneko et al. (2012), who found no changes in von Willebrand factor levels and factor VIII levels $3 \mathrm{~h}$ after hemorrhagic shock among the normal saline, low-HES, and medium-HES groups in hemorrhagic shock rats.

The reason that the coagulation system was not affected was that these animals had been retransfused to $>70 \%$ of the shed blood (whole blood containing large amounts of coagulation factors) before $\mathrm{R} 4$ to reach the $\mathrm{Hb}$ goal. Second, the coagulation system is significantly less impaired when infusing 6\% HES 130/0.4 combined with RL than with HES alone (Moretti et al., 2003). Third, as a medium-weight HES solution, HES 130/0.4 impairs the coagulation system less than higher-molecular-weight HES solutions (Langeron et al., 2001).

However, our study has some limitations. As with any animal model, the data cannot be directly extrapolated to human models. Nonetheless, the principal advantage of an animal model is that it ensures homogeneity and a controlled environment for the standardization of the evaluated interventions, permits measurements at defined time points, and removes confounding factors common to clinical trials. Another limitation of the present study is that thromboelastometry was not used in the measurement of coagulation parameters; therefore, the effects of the tested solutions on coagulation parameters remains to be conclusively elucidated.

The main findings of our randomized controlled experimental study are summarized as follows: 1) in this canine hemorrhagic shock model, the 3:1 group received greater LR (crystalloid) amounts and showed larger EVLWI than the 1:3 group. This increase EVLW may explain the consequent hypoxemia; 2) the $1: 3$ and $1: 2$ groups received greater amounts of HES (colloid containing $0.9 \% \mathrm{NaCl}$ ), which resulted in hypernatremia, hyperchloremia, and hyperchloremic acidosis; 3) goal-directed fluid therapy with LR and HES at 1:2 and 1:3 ratios in this canine hemorrhagic shock model may impair renal function. 
In view of these results, we suggest that infusing resuscitation fluids with an LR:HES ratio of 3:1 in the setting of hemorrhagic shock for $24 \mathrm{~h}$ from the onset of shock may be associated with EVLW increase and hypoxemia, whereas rating fluids at ratios of 1:3 and 1:2 may result in hypernatremia, hyperchloremia, hyperchloremic acidosis, and impaired renal function. Therefore, fluids with LR: HES ratios of 2:1 and 1:1 for resuscitation of hemorrhagic shock in canines may be superior to fluids with LR:HES ratios of 3: 1, 1:2 and 1:3 with better acid-base, electrolyte, EVLW, and renal function profiles.

\section{ACKNOWLEDGMENTS}

The authors are indebted to Wei-Qi Wang, Zi-Chao Dai, Rong-Qiang Yang, Li-Ling Chen, Gui-Fang Sun, Feng Guo, Lin-Xu Shi, Qun Xian, and Jun Li for their excellent technical support, and to Li-Ping $\mathrm{He}, \mathrm{PhD}$, for statistical advice. Thanks also go to Dr. Loh Cheng for revision of the articles.

Research supported by the National Natural Science Foundation of China (\#81060033); the Provincial Scientific Research Foundation of Education Department of Yunnan Province, (\#2010Z030); the Yunnan Natural Science Foundation (\#2009CD176); and the Yunnan Clinical Research Fund (\#D-201213).

\section{REFERENCES}

Almaca E, Aksua U, Bezemera R, Jong W, et al. (2012). The acute effects of acetate-balanced colloid and crystalloid resuscitation on renal oxygenation in a rat model of hemorrhagic shock. Resuscitation 83: 1166-1172.

Brill SA, Stewart TR, Brundage SI and Schreiber MA (2002). Base deficit does not predict mortality when secondary to hyperchloremic acidosis. Shock 17: 459-462.

Cabrales P, Tsai AG and Intaglietta M (2007). Resuscitation from hemorrhagic shock with hydroxyethyl starch and coagulation changes. Shock 28: 461-467.

Ertmer C, Kampmeier T, Rehberg S and Lange M (2011). Fluid resuscitation in multiple trauma patients. Curr. Opin. Anesthesiol. 24: 202-208.

European Medicines Agency (2013). PRAC recommends suspending marketing authorisations for infusion solutions containing hydroxyethyl-starch. Available at [http://www.ema.europa.eu/ema/index.jsp?curl=pages/news_and events/news/2013/06/news_detail_001814.jsp\&mid=WC0b01ac058004d5c1].

Feldheiser A, Pavlova V, Bonomo T, Jones, A, et al. (2013). Balanced crystalloid compared with balanced colloid solution using a goal-directed haemodynamic algorithm. Br. J. Anesth. 110: 231-240.

Fries D, Innerhofer P, Klingler A, Berresheim U, et al. (2002). The effect of the combined administration of colloids and lactated Ringer's solution on the coagulation system: an in vitro study using thrombelastograph coagulation analysis (ROTEG). Anesth. Analg. 94: 1280-1287.

Guyton AC, Jones CE and Coleman TG (1973). Normal Cardiac Output and Its Variation. In: Cardiac Output and Its Regulation (Guyton AC, Jones CE and Coleman TG, eds.). WB Saunders Co., Philadelphia, 3-29.

Haas T, Fries D, Holz C, Innerhofer P, et al. (2008). Less impairment of hemostasis and reduced blood loss in pigs after resuscitation from hemorrhagic shock using the small-volume concept with hypertonic saline/hydroxyethyl starch as compared to administration of 4\% gelatin or 6\% hydroxyethyl starch solution. Anesth. Analg. 106: 1078-1078.

Haisch G, Boldt J, Krebs C, Kumle B, et al. (2001). The influence of intravascular volume therapy with a new hydroxyethyl starch preparation (6\% HES 130/0.4) on coagulation in patients undergoing major abdominal surgery. Anesth. Analg. 92: 565-571.

Holcomb JB, McMullin NR, Pearse L, Caruso J, et al. (2007). Causes of death in U.S. special operations forces in the global war on terrorism: 2001-2004. Ann. Surg. 245: 986-991.

Kimberger O, Amberger M, Brandt S, Plock J, et al. (2009). Goal-directed colloid administration improves the microcirculation of healthy and perianastomotic colon. Anesthesiology 110: 496-504.

Lang K, Boldt J, Suttner S and Haisch G (2001). Colloids versus crystalloids and tissue oxygen tension in patients undergoing major abdominal surgery. Anesth. Analg. 93: 405-409. 
Langeron O, Doelberg M, Ang ET, Bonnet F, et al. (2001). Voluven, a lower substituted novel hydroxyethyl starch (HES 130/0.4), causes fewer effects on coagulation in major orthopedic surgery than HES 200/0.5. Anesth. Analg. 92: 855-865.

Mauch J, Madjdpour C, Kutter AP, Spielmann N, et al. (2012). Effect of rapid fluid resuscitation using crystalloids or colloids on hemostasis in piglets. Paediatr. Anaesth. 23: 258-264.

Moretti EW, Robertson KM, El-Moalem H and Gan TJ (2003). Intraoperative colloid administration reduces postoperative nausea and vomiting and improves postoperative outcomes compared with crystalloid administration. Anesth. Analg. 96: 611-617.

Myburgh JA, Finfer S, Bellomo R, Billot L, et al. (2012). Hydroxyethyl starch or saline for fluid resuscitation in intensive care. N. Engl. J. Med. 367: 1901-1911.

Pearse R, Dawson D, Fawcett J, Rhodes A, et al. (2005). Early goal-directed therapy after major surgery reduces complications and duration of hospital stay. A randomized, controlled trial. Crit. Care. 9: 687-693.

Perner A, Haase N, Guttormsen AB, Tenhunen J, et al. (2012). Hydroxyethyl starch 130/0.42 versus Ringer's acetate in severe sepsis. N. Engl. J. Med. 367: 124-134.

Phillips CR, Vinecore K, Hagg DS, Sawai RS, et al. (2009). Resuscitation of hemorrhagic shock with normal saline vs lactated Ringer's: effects on oxygenation, extravascular lung water and hemodynamics. Crit. Care 13: R30.

Scheeren TW, Wiesenack C, Gerlach H and Marx G (2013). Goal-directed intraoperative fluid therapy guided by stroke volume and its variation in high-risk surgical patients: a prospective randomized multicentre study. J. Clin. Monit. Comput. 27: 225-233.

Scheingraber S, Rehm M, Sehmisch C and Finsterer U (1999). Rapid saline infusion produces hyperchloremic acidosis in patients undergoing gynecologic surgery. Anesthesiology 90: 1265-1270.

Walsh SR, Cook EJ, Bentley R, Farooq N, et al. (2008). Perioperative fluid management: prospective audit. Int. J. Clin. Pract. 62: 492-497.

Warren BB and Durieux ME (1997). Hydroxyethyl starch: safe or not? Anesth. Analg. 84: 206-212.

Wilcox CS (1983). Regulation of renal blood flow by plasma chloride. J. Clin. Invest. 71: 726-735.

Williams EL, Hildebrand KL, McCormick SA and Bedel MJ (1999). The effect of intravenous lactated ringers solution versus $0.9 \%$ sodium chloride on serum osmolarity in human volunteers. Anesth. Analg. 88: 999-1003.

Yoneko M, Suzuki Y and Kojima J (2012). Comparative effects of low and medium molecular hydroxyethyl starch (HES) solutions on hemorrhagic shock in rats. Masui 61: 57-63. 